\title{
La dimensión argumentativa y tecnológica en la formación de docentes de ciencias
}

\author{
Argumentative and technological dimensions \\ in science teacher training
}

\author{
Leticia Garcia Romano, ${ }^{1,2}$ María Eugenia Condat ${ }^{1}$. \\ Maricel Occelli ${ }^{1,2}$. Nora Valeiras ${ }^{1}$
}

\begin{abstract}
Resumen: El presente artículo analiza las nociones de argumentación de docentes de ciencias y estudiantes de escuela secundaria y profundiza dicho análisis a través de la caracterización de la potencialidad argumentativa de tres recursos tecnológicos utilizados en el marco de la formación inicial y continua de profesores de ciencias. Los recursos estudiados son los siguientes: foros de debate, mapas de argumentos (Debategraph) y WISE (Web-based Inquiry Science Environment). En lo que respecta a las ideas sobre argumentación de profesores y estudiantes, los resultados muestran una diversidad de posturas, expectativas y habilidades. En lo que se refiere a los recursos tecnológicos, puede decirse que los tres recursos combinan dos aspectos clave para el aprendizaje de las ciencias: la argumentación y la colaboración. En función de los resultados obtenidos, se proponen sugerencias para la incorporación de la dimensión argumentativa y tecnológica en la formación de docentes de ciencias.
\end{abstract}

Palabras-clave: Argumentación. Tecnologías de la información y la comunicación. Formación docente.

\begin{abstract}
We discuss in this paper the notions of argumentation held by science teachers and high school students. The analysis is taken to a deeper level through the characterization of three technological resources that are employed in the context of initial and continuous training of science teachers. The resources studied are: discussion forums, maps of arguments (Debategraph) and WISE (Web-based Inquiry Science Environment). With regard to the ideas on argumentation of teachers and students, the results show a variety of positions, expectations and skills. When it comes to technology resources, one can say that the three resources combine two key aspects in science learning: argumentation and collaboration. Based on the results obtained, suggestions for incorporating argumentative and technological dimensions in science teacher training are proposed.
\end{abstract}

Keywords: Argumentation. Information and communication technologies. Teacher training.

\footnotetext{
${ }^{1}$ Universidad Nacional de Córdoba, Facultad de Ciencias Exactas, Físicas y Naturales, Departamento de Enseñanza de la Ciencia y la Tecnología, Córdoba, Argentina. E-mail: <lgarciaromano@gmail.com>.

${ }^{2}$ Consejo Nacional de Investigaciones Científicas y Técnicas (CONICET), Argentina.
} 


\section{Introducción}

La argumentación es un proceso clave para el aprendizaje dado que sus propiedades semióticas y dialógicas convierten a esta actividad discursiva en un recurso privilegiado de mediación en los procesos de construcción de conocimiento (LEITÃO, 2007).

En lo que se refiere al campo de la didáctica de las ciencias, el interés por el desarrollo de procesos argumentativos por parte de los alumnos ha ido en aumento en los últimos 15 años (DRIVER; NEWTON; OSBORNE, 2000; JIMÉNEZ-ALEIXANDRE, 2010; JIMÉNEZ-ALEIXANDRE; ERDURAN, 2008; KELLY; BAZERMAN, 2003). En este marco, Adúriz-Bravo (2014) caracteriza tres formas distintivas a través de las cuales se ha justificado la importancia de la argumentación en clases de ciencias. En primer lugar, se sostiene la idea de que aprender a argumentar es un proceso central para aprender a pensar y construir nuevos conocimientos. En segundo lugar, se considera que el hecho de apropiarse de una práctica científica como la argumentación aporta a la construcción de una idea de ciencia coherente con la contribución de la filosofía e historia de la ciencia. Por último, se subraya el rol de la argumentación en la alfabetización científica, apuntando a la posibilidad de que los estudiantes participen en debates socio-científicos.

En esta dirección, y con relación a las dos primeras formas de aproximación a la inclusión de la argumentación mencionadas previamente, la función de los docentes no se basaría sólo en la devolución crítica respecto de los argumentos de los estudiantes, sino también en gestionar los saberes y actividades en construcción, orientándolos hacia afirmaciones y procedimientos estabilizados, admitidos en las comunidades de expertos en un área de estudio (BUTY; PLANTIN, 2008).

En lo que atañe a la tercera forma, los docentes deberían tener en cuenta las dificultades inherentes a la integración de distintos campos disciplinares en un debate socio-científico, diseñando actividades que tiendan a valorar la mayor cantidad de ventajas y desventajas sobre un tema, sin caer en oposiciones simplistas (JIMÉNEZ-ALEIXANDRE, 2010).

$\mathrm{Al}$ analizar las tres formas de manera conjunta, la enseñanza de la argumentación se encuentra ligada a un aspecto central de la formación de docentes en ciencias, ya que se vincula a una pedagogía basada en la construcción del conocimiento y no a una instrucción basada en su transmisión (SANDOVAL; MILLWOOD, 2008).

Sin embargo, tal como señala Plantin (2014), existe una gran carencia en la formación teórica y práctica de los profesores de ciencias en lo que refiere a la argumentación. Una forma de mejorar dichas instancias de formación podría ser la implementación de programas que presten atención a los conocimientos y las creencias que poseen los profesores sobre la enseñanza de la argumentación (ZOHAR, 2008) y a lo que los propios estudiantes conocen respecto del proceso argumentativo en ciencias (REVEL CHION; MEINARDI; ADÚRIZ-BRAVO, 2014).

En función de estos antecedentes, se considera que la investigación educativa relacionada con el aprendizaje y las concepciones acerca de la argumentación por parte de docentes y estudiantes - destacada como área de vacancia por una serie de autores (ARCHILA, 2012; JIMÉNEZ-ALEIXANDRE; ERDURAN, 2015) - puede constituir un aporte significativo al campo de trabajo.

Por otra parte, diversas investigaciones plantean el hecho de que las Tecnologías de la Información y la Comunicación (TIC) favorecen el desarrollo de habilidades para la interpretación de textos, la representación gráfica, el aprendizaje colaborativo y el aprendizaje de 
conceptos complejos a través del modelado y la simulación (DORI; SASSON, 2008; SOLANO ARAUJO; VEIT; MOREIRA, 2008; VÁZQUEZ-ABAD et al., 2004). Además, los ambientes enriquecidos con TIC fomentan la expresión de las voces que no suelen manifestarse en otros ámbitos, facilitan el aprendizaje a través de la argumentación escrita y favorecen la ocurrencia de diálogos epistémicos que incluyen la argumentación (CLARK et al., 2008; DE VRIES; LUND; BAKER, 2002; LUEHMANN; FRINK, 2012).

Así, muchos entornos tecnológicos ofrecen posibilidades de aprender a través del desarrollo de prácticas argumentativas y, tal como señalan Clark et al. (2008), los beneficios potenciales de dichas herramientas no se limitan a los estudiantes sino que pueden extenderse a los profesores, modelando sus propias argumentaciones y ayudándolos a interpretar los desarrollos argumentativos de sus estudiantes.

Sin embargo, las opiniones respecto de las TIC son amplias: algunas vinculadas a una visión optimista sobre su integración en la docencia y otras, más pesimistas, relacionadas con los problemas que implican, entre ellos, la falta de formación específica de los docentes (PAREDES; ARRUDA, 2012). En el campo particular de la formación de profesores en Argentina, tanto el Plan Nacional de Educación Obligatoria y Formación Docente como los lineamientos básicos sobre formación docente de profesores universitarios elaborados por la comisión mixta de la Asociación Nacional de Facultades de Humanidades y Educación (ANFHE) y el Consejo Universitario de Ciencias Exactas y Naturales (CUCEN) proponen el desarrollo de acciones formativas mediadas por TIC en la enseñanza de las disciplinas en la formación docente, y trazan un lugar importante para las habilidades discursivas/comunicativas y la producción y el análisis de textos (ARGENTINA, 2012; LINEAMIENTOS..., 2011).

Sin embargo, también en el contexto argentino, encontramos estudios que no sólo describen la escasez de habilidades argumentativas de los futuros docentes de ciencias en el transcurso de su formación inicial (CAMPANER, 2008) sino que además ponen en evidencia que los docentes despliegan estrategias que implican la utilización de tecnologías en sus modalidades más instrumentales (GARCIA ROMANO et al., 2015; OCCELLI; GARCIA; MASULLO, 2012).

Además de esto, el estudio de ambas dimensiones es particularmente complejo debido a las diferentes concepciones respecto de lo que implica argumentar (PLANTIN, 2014) y al hecho de que "las tecnologías son significadas, usadas y apropiadas de diferentes modos por los actores de la comunidad escolar" (ZANNOTTI; ARANA, 2015, p. 139).

En consideración de estas cuestiones, el presente artículo se propone un doble objetivo: por una parte, analizar las nociones de argumentación de docentes de ciencias y estudiantes de escuela secundaria, y, por otra, profundizar dicho análisis a través de la caracterización de la potencialidad argumentativa de tres recursos tecnológicos utilizados en el marco de la formación docente inicial y continua de profesores de ciencias.

\section{Consideraciones metodológicas}

El estudio de las concepciones acerca de la argumentación se realizó desde una perspectiva esencialmente fenomenográfica. Este enfoque parte de la idea de que las personas perciben, conceptualizan y entienden sus experiencias - y las dimensiones que las componen - de formas cualitativamente diferentes (MARTON, 1981; MATEOS; SOLÉ, 2012). 
Se desarrolló una indagación exploratoria con un cuestionario que fue respondido por 25 profesores de ciencias en activo. A través de este instrumento, se preguntó a los docentes qué significaba para ellos defender un punto de vista en ciencias naturales, pudiendo marcar como respuesta una o más de las siguientes opciones: (a) justificar desde lo que se sabe, sin buscar información; (b) justificar e intentar convencer a los demás de que se trata del punto de vista correcto; (c) justificar sin intenciones de convencer a otros; (d) presentar pruebas que lo avalen. Asimismo, se solicitó - a través de preguntas abiertas - que describieran si creían que existían temas/contenidos con los que fuera más factible trabajar la argumentación en sus clases, que fundamentaran por qué sostenían esa idea, y que indicaran las dificultades que encontraban al enseñar a argumentar en sus clases.

Además de esto, se realizaron entrevistas en profundidad a dos docentes de ciencias. Las preguntas-guía que regularon dichas instancias fueron las siguientes:

1 ¿Qué implica argumentar en clases de ciencias?

2 ¿Piensa que es posible trabajar destrezas relacionadas con la argumentación en las clases de ciencias?

3 ¿Cree que existen temas particulares con los que es más factible trabajar la argumentación?

4 ¿Qué papel/rol/función piensa que tienen el uso y la búsqueda de evidencias y pruebas para defender o justificar una posición en ciencias?

Por último, se analizaron las creencias de los estudiantes. Por un lado, se solicitó la construcción de una expresión metafórica a 400 alumnos de escuela secundaria. En el marco de sus clases de ciencias, los estudiantes completaron la siguiente frase: "Argumentar en clases de ciencias es como...". Esta propuesta deriva del trabajo de Lakoff y Johnson (1980), quienes consideran que las metáforas son parte integral de nuestro pensamiento y lenguaje, permiten entender nuestro mundo de manera que otros modos de pensamiento no podrían, y desempeñan un rol central en la forma en la que los miembros de una cultura conceptualizan su experiencia. Asimismo, se tomaron como referencia metodológica una serie de investigaciones que recurren a la construcción de metáforas como una forma de acceso al sistema de concepciones de los estudiantes (ARMSTRONG, 2008; LEVIN; WAGNER, 2006; PEREIRA; DI STEFANO, 2007). Por otro lado, y de manera similar a lo realizado con los docentes, se encuestó a 149 estudiantes de escuela secundaria respecto de lo que significa para ellos defender un punto de vista en ciencias (pregunta cerrada con las mismas opciones de respuesta que el cuestionario para profesores), y sobre su opinión acerca de la importancia que los docentes atribuyen a la realización de diferentes tareas vinculadas a la argumentación en las clases de ciencias: (a) buscar distintas fuentes de información para justificar sus respuestas; (b) usar la información presente en libros de texto, en fotocopias proporcionadas por el/la profesor/a o en los apuntes de clases para justificar sus respuestas; (c) participar en debates en los que se discutan diferentes posturas; (d) participar en debates en los que se defienda una sola postura (pregunta cerrada con escala de cuatro puntos: "muy importante", "importante", "poco importante" y "nada importante").

En lo que respecta a las propuestas de formación docente mediada por TIC, se analiza el potencial argumentativo de tres recursos tecnológicos: foros de debate, mapas de argumentos (Debategraph) y WISE (Web-based Inquiry Science Environment). Los foros de debate fueron uno de los elementos centrales de un curso de formación docente continua que tomó como eje una temática socio-científica y en el cual participaron 96 profesores de Biología y Química de 
nivel secundario y universitario. Los mapas de argumentos se implementaron en dos instancias: por un lado, se utilizaron en una propuesta de trabajo con 37 estudiantes en el primer tramo de su formación inicial como profesores de Biología y, por otro lado, se recurrió a ellos para representar gráficamente las discusiones mantenidas en los foros de debate del curso de formación para profesores de Biología y Química mencionado precedentemente. El entorno WISE fue el centro de una actividad de investigación implementada con dos estudiantes avanzados de un profesorado de Biología.

Tanto el análisis de las creencias de docentes y estudiantes como la indagación acerca de las potencialidades y las nociones de argumentación subyacentes a los tres recursos tecnológicos utilizados en la formación docente fueron realizados tomando como referencia las categorías teóricas propuestas por diversos autores en el área de trabajo. Así, se analizó la presencia de ideas ligadas a la argumentación como explicación y como justificación (ADÚRIZ-BRAVO, 2014); se estudió si la argumentación se presenta como un debate entre proponentes y oponentes (PLANTIN, 1998), como la resolución de diferencias de opinión entre personas (VAN EEMEREN; GROOTENDORST; HENKEMANS, 2006) o como la evaluación de enunciados a la luz de las pruebas (JIMÉNEZ-ALEIXANDRE, 2010); se caracterizó la presencia de ideas vinculadas a la dimensión epistémica de la argumentación (LEITÃO, 2007); y se indagó la relación entre la posibilidad de argumentar y el aprendizaje colaborativo (CLARK et al., 2008).

\section{¿Qué piensan los docentes sobre la argumentación?}

En lo que respecta a la indagación exploratoria realizada con 25 profesores de ciencias en ejercicio, se encontró que un $72 \%$ sostuvo que defender un punto de vista en ciencias naturales significa "presentar pruebas que lo avalen" y un $28 \%$ se refirió a la idea de "justificar sin intenciones de convencer a otros". Cabe aclarar que ninguno de los profesores optó por la idea de "justificar e intentar convencer a los demás de que se trata del punto de vista correcto", premisa que explicita el componente persuasivo de la argumentación, ni por la noción de “justificar desde lo que se sabe, sin buscar información”.

Las premisas elegidas se centran en la explicación y, en consecuencia, se caracterizan por una argumentación gestada de manera independiente de las audiencias (ADÚRIZ-BRAVO, 2014). Retomando el trabajo de Plantin (2004), se sostiene que una de las causas vinculadas a estas concepciones puede ser el componente de agresividad que suele atribuirse a la idea de debatir. Desde esa creencia, la argumentación podría ser el punto de partida para la ruptura de relaciones entre alumnos, cuestión que los docentes no querrían provocar.

Respecto de si existen temas o contenidos con los que es más factible trabajar la argumentación en clase, se encontró que la mayoría de los profesores alude a cuestiones socio-científicas $(60 \%)$, que se expresan en estas palabras:

Profesor $\mathrm{N}^{\circ}$ 9: Sí, discusiones éticas sobre fertilización asistida [...].

Profesor $\mathrm{N}^{\circ}$ 19: Presentación de la biodiversidad. El agua, cuidado y problemas futuros. [...]. 
La posibilidad de que los docentes respondan abiertamente a las preguntas planteadas permite decir que las respuestas dadas a la pregunta cerrada no implican necesariamente que los docentes se asienten más en una perspectiva monologal que en una noción dialógica de la argumentación (PLANTIN, 2012). Sin embargo, se trata de una idea de lo dialógico centrada en la posibilidad de ampliar la mirada respecto de un tema, valorar otros puntos de vista y fomentar el pensamiento crítico, sin aludir a la idea de persuasión:

Profesor $\mathrm{N}^{\circ}$ 5: Propender a efectivizar una reflexión crítica. Valorar los demás puntos de vista. Enriquecer los puntos de vista individuales.

Profesor $\mathrm{N}^{\circ}$ 14: Pienso que [la argumentación] es sumamente importante para conocer los diferentes puntos de vista y que se puedan replantear las decisiones que toman día a día.

Por último, resulta relevante mencionar que los profesores encuentran dificultades asociadas a la enseñanza de la argumentación, principalmente centradas en la predisposición de los alumnos, ya sea porque consideran que los estudiantes no se comprometen con una actividad cognitiva desafiante como la argumentación, pierden el foco del debate o prefieren utilizar recursos tecnológicos en lugar de debatir oralmente o por escrito:

\footnotetext{
Profesor $\mathrm{N}^{\circ}$ 6: Los alumnos están acostumbrados a un aprendizaje 'memorístico', sin tomar partido....

Profesor $\mathrm{N}^{\circ}$ 4: [una de las dificultades para enseñar a argumentar es la] desviación del tema original.

Profesor $\mathrm{N}^{\mathrm{o}}$ 1: Los alumnos lo ven poco atractivo [al aprendizaje de la argumentación], prefieren utilizar otro tipo de enseñanza. Prefieren utilizar las netbooks, cmap, power point para exponer un tema o clases expositivas.
}

Así, se observa cómo la posibilidad de argumentar en el aula interpela la forma en que pensamos la enseñanza, pero también es llamativo el hecho de que se instala el foco de atención en las aparentes deficiencias y preferencias de los estudiantes, pensándolas más como obstáculos que como posibilidades de afrontar la práctica de enseñar de una forma diferente.

En esta dirección, pero en lo inherente a las entrevistas, el análisis de las respuestas de los profesores permitió caracterizar dos posicionamientos polares: uno que establece a la argumentación como un conocimiento del docente y con base en una serie de recursos, y otro que relaciona la argumentación con las habilidades y dificultades de los estudiantes (Cuadro 1). 
Cuadro 1. Caracterización de dos posicionamientos docentes contrapuestos respecto de la argumentación en ciencias

\begin{tabular}{|l|l|l|}
\hline \multicolumn{1}{|c|}{$\begin{array}{c}\text { Posicionamiento centrado } \\
\text { en el docente y los recursos }\end{array}$} & \multicolumn{1}{|c|}{$\begin{array}{c}\text { Posicionamiento centrado } \\
\text { en los alumnos }\end{array}$} \\
\hline $\begin{array}{l}\text { Argumentar en clases } \\
\text { de ciencias es... }\end{array}$ & $\begin{array}{l}\text { Tomar una posición y transmitírsela a } \\
\text { los estudiantes. }\end{array}$ & $\begin{array}{l}\text { Explicar y fundamentar. Lo hacen } \\
\text { todos los alumnos, todo el tiempo. }\end{array}$ \\
\hline $\begin{array}{l}\text { Uso de pruebas y } \\
\text { evidencias }\end{array}$ & $\begin{array}{l}\text { Usar evidencias permite confirmar la } \\
\text { veracidad de los conocimientos que } \\
\text { se le van a presentar a los alumnos. }\end{array}$ & $\begin{array}{l}\text { Los alumnos recurren al uso de } \\
\text { pruebas y evidencias para defender } \\
\text { una postura. }\end{array}$ \\
\hline $\begin{array}{l}\text { Dificultades para } \\
\text { argumentar }\end{array}$ & $\begin{array}{l}\text { Los problemas para argumentar } \\
\text { se asocian a la falta de recursos } \\
\text { materiales ya que sólo se relaciona } \\
\text { la argumentación con el trabajo de } \\
\text { laboratorio y las salidas de campo. }\end{array}$ & $\begin{array}{l}\text { Los alumnos carecen de muchas } \\
\text { habilidades argumentativas; es } \\
\text { necesario que los profesores les } \\
\text { enseñen a argumentar. }\end{array}$ \\
\hline
\end{tabular}

Fuente: elaborado por las autoras.

El posicionamiento centrado en el docente y los recursos puede relacionarse con modelos más transmisivos, mientras que el posicionamiento con base en los alumnos puede vincularse a posturas más constructivas acerca de lo que significa enseñar y aprender. Tomando en consideración las nociones desarrolladas por Plantin (2014), la primera idea se refiere a un profesor que quiere cambiar las representaciones y opiniones que sus estudiantes tienen sobre el conocimiento científico, mientras que la segunda se orienta hacia la participación activa del alumnado, recuperando su voz en las instancias argumentativas. Asimismo, en el primer caso, la idea de argumentación se vincula sólo a determinadas prácticas consideradas científicas -trabajo de laboratorio y de campo- y no al dominio amplio de estrategias didácticas factibles de implementar en la enseñanza de contenidos científicos.

\section{¿Qué piensan los estudiantes sobre la argumentación?}

Con el objetivo de conocer las creencias de los alumnos, se procedió a analizar las ideas de argumentación que aparecían en las metáforas construidas. El análisis de las respuestas condujo al establecimiento de siete dimensiones principales, tal como se observa en el Cuadro 2. 
Cuadro 2. Concepciones sobre la argumentación de estudiantes de escuela secundaria

\begin{tabular}{|l|l|}
\hline \multicolumn{1}{|c|}{ Dimensión } & \multicolumn{1}{c|}{ Respuesta de los estudiantes } \\
\hline $\begin{array}{l}\text { (Trans)formación del } \\
\text { conocimiento }\end{array}$ & $\begin{array}{l}\text { Entrar en un laberinto sin salida que te mezcla; hasta que encuentras la saliday } \\
\text { entiendes todo. } \\
\text { Que aprendes más de lo que sabias. }\end{array}$ \\
\hline $\begin{array}{l}\text { Alfabetización } \\
\text { científica }\end{array}$ & $\begin{array}{l}\text { El aprendizaje de nuevos contenidos que ayuden en la vida cotidiana. } \\
\text { Ser científico. }\end{array}$ \\
\hline Dificultad & $\begin{array}{l}\text { Explicarle a un bebé cómo fue el origen de la bumanidad (no entendería nada). } \\
\text { Nadar en un mar de engrudo [....]. }\end{array}$ \\
\hline $\begin{array}{l}\text { Expresión de } \\
\text { opiniones }\end{array}$ & $\begin{array}{l}\text { Hacer un debate donde cada uno da su propia opinión sobre el tema. } \\
\text { Expresar nuestros puntos de vista }\end{array}$ \\
\hline Incertidumbre & Mezclar ingredientes sin saber cuál será el resultado. \\
\hline Modos de argumentar & Cocinar, hay distintas maneras. \\
\hline Persuasión & Cuando una novia te convence. \\
\hline
\end{tabular}

Fuente: elaborado por las autoras.

Estas ideas recuerdan en un punto lo expuesto por Scardamalia y Bereiter (1992), quienes caracterizan las diferencias entre "decir el conocimiento" y "transformar el conocimiento". En otras palabras, y tomando como base lo propuesto por Carlino (2002), la argumentación es conceptualizada a partir de su potencial epistémico. Sin embargo, tal como sucede para el caso de la lectura y la escritura, la argumentación también es concebida como un campo de dificultades, incertidumbres y, en consecuencia, de posibles desencuentros de ideas entre profesores y estudiantes, y entre los propios alumnos. Además, aparecen tanto la noción lógica como dialógica de la argumentación (ADÚRIZ-BRAVO, 2014) y se vislumbra el vínculo entre el discurso argumentativo y la alfabetización científica (LARRAÍN, 2009).

A diferencia de lo encontrado en los docentes, en el caso de los alumnos, un 44\% sostuvo que defender un punto de vista en ciencias naturales significa "presentar pruebas que lo avalen", un 34\% optó por la idea de que implica "justificar e intentar convencer a los demás de que se trata del punto de vista correcto", un $22 \%$ expresó que se trata de "justificar desde lo que se sabe, sin buscar información”, y un 17\% se refirió a la idea de "justificar sin intenciones de convencer a otros". Este panorama más diverso puede deberse a la variedad de tareas que realizan los estudiantes con diferentes profesores, lo que conlleva a transitar la argumentación desde diferentes posturas.

Finalmente, en lo que respecta a la importancia que los alumnos atribuyen a diferentes tareas propuestas por los docentes en sus clases de ciencias, el 77\% indicó como importante o muy importante el hecho de "buscar distintas fuentes de información para justificar sus res- 
puestas" y el $81 \%$ la idea de "usar la información presente en libros de texto, en fotocopias proporcionadas por el/la profesor/a o en los apuntes de clases para justificar sus respuestas". Estos porcentajes disminuyeron al 52\% cuando se preguntó acerca de la importancia de "participar en debates en los que se discutan diferentes posturas" y a un $40 \%$ al indagar respecto de la relevancia de "participar en debates en los que se defienda una sola postura". Estas respuestas nos acercan más al posible uso de estrategias tradicionales de enseñanza, centradas en una modalidad de un docente que pregunta y un alumno que responde con base en la lectura de textos, y no tanto a la posibilidad de un contexto general de debate, en el que los alumnos argumentan teniendo en cuenta audiencias más amplias que el propio docente y donde tanto las preguntas como las respuestas, los argumentos y los contra-argumentos sean construidos activamente por docentes y alumnos.

En resumen, estos hallazgos permiten mostrar la complejidad del campo de la argumentación en clases de ciencias, caracterizada por la diversidad de posturas, expectativas y habilidades, lo que, sin el desarrollo de actividades específicas y el despliegue de dispositivos didácticos particulares, puede transformar las prácticas argumentativas en instancias desmotivadoras, frustrantes y alejadas de las prácticas científicas.

\section{El aporte de las tecnologías a las prácticas argumentativas desarrolladas en la formación del profesorado}

Tal como se señala en la introducción de este artículo, muchos entornos tecnológicos ofrecen posibilidades de aprender a través del desarrollo de prácticas argumentativas. En función de esto y de los resultados presentados en el apartado anterior, desde el año 2009, hemos implementado estrategias específicas tendientes a formar a los profesores de ciencias en argumentación, valiéndonos de las oportunidades que ofrecen las TIC en este sentido. A continuación, se analizarán las principales características de tres recursos tecnológicos utilizados, destacando la noción de argumentación que subyace a las propuestas y su potencial para la argumentación en el marco de la formación del profesorado.

\section{Foros}

Los "foros" o "foros de debate en línea" son recursos incluidos en distintos entornos mediados por tecnología. En concordancia con investigaciones realizadas en otros países, hemos encontrado que los estudiantes señalan que la participación en un contexto virtual mediado por foros implica poner mayor atención a la forma de escribir y expresar ideas en comparación con lo que sucede en un contexto presencial (GARCIA ROMANO; VALEIRAS, 2011).

Ahora bien, ¿cómo podemos caracterizar los foros desde un punto de vista argumentativo? Por un lado, pueden ser pensados desde la lógica de un debate entre proponentes y oponentes (PLANTIN, 1998). Sin embargo, también pueden concebirse como soporte para la resolución de diferencias de opinión entre personas (VAN EEMEREN; GROOTENDORST; HENKEMANS, 2006).

Desde una perspectiva que combina ambas nociones, utilizamos foros para pensar alternativas de solución a una problemática socio-científica en el marco de un seminario de 
formación permanente del profesorado. Los principales resultados, destacables desde el punto de vista de la argumentación, fueron los siguientes:

En primer lugar, es importante señalar que los participantes planificaron y revisaron sus textos argumentativos colaborativamente. El Cuadro 3 presenta intercambios realizados en el foro que ejemplifican esta situación.

Cuadro 3. Intercambios escritos del foro orientados a la planificación y la revisión textual

Hola Isabel: Por lo que dice la consigna de la actividad [...] yo coincido con vos en que el texto que [...] armemos puede estar orientado a responder que el origen de las malformaciones está en los agroquímicos y no en los transgénicos. (Mar).

...Supongo que en base a lo que vamos a leer, redefiniremos el problema y armaremos la redacción. Coincido en que el problema debe estar en la exposición a los agroquimicos a la que conllevan los OMG. (Gimena).

Intercambian mensajes de organización de tareas y mensajes afectivos que hacen a la cohesión grupal

Chicas: Yo escribi algo en la wiki, me gustaría que me den su parecer al respecto. Quedo a la espera de sus opiniones. Cariños. (Isabel).

Isabel: Me pareció muy bueno, concreto y preciso el texto, no creo necesario modificar nada. (Mar).

Intercambian nuevamente mensajes de organización de tareas y algunos en los que explican por qué agregaron determinada información en el texto que están construyendo en la Wiki como se ejemplifica en los siguientes mensajes:

Chicas agrego en Wiki puntos que me parecieron interesantes del articulo 'Impacto de plaguicidas'. También me pareció muy interesante (pero no lo agregaré en wiki, porque nos vamos a exceder en artículos) un documento de San Francisco provincia de Córdoba donde se aprobó una Ley de Agroquimicos (Ordenanza No 5531)... (Haydee).

Hola chicas: Ya subi a wiki el texto que armé, me pareció un poco extenso, pero todo para mí era importante, que no se podía obviar, ustedes fijensé, modifiquen arreglen... (Mar).

Fuente: elaborado por las autoras.

En segundo lugar, el espacio del foro resultó fructífero para que los participantes construyeran preguntas sobre un tema e interrogaran a los textos de referencia:

He leido con detenimiento el artículo aportado por Pablo y parece estar muy bueno y responder un sinnúmero de dudas que todos nos hemos planteado alguna vez: Sin embargo, en todas las respuestas siempre permanece un cierto grado de incertidumbre [...] Les pregunto: ¿Qué opinarian de la transgénesis si se experimentara con humanos en beneficio de las plantas, bacterias, etc? ¿Quiénes creemos ser para determinar que una 
especie de insectos es una plaga? ¿Tenemos más derechos que el resto de los seres vivos para determinar su futuro? [...] (Benjamín).

Finalmente, tal como se describiera en Garcia Romano y Occelli (2012), es necesario resaltar que la tendencia de los grupos colaborativos hacia el debate o hacia el consejo tuvo relación con los puntos de vista de los participantes al inicio del curso. Así, la indagación sistemática de los puntos de vista iniciales puede resultar muy útil al momento de organizar los grupos de trabajo colaborativos, monitorear los debates posteriores y generar preguntas que acerquen a los participantes a ideas que no explicitan en los foros sin la intervención de sus tutores.

\section{Mapas de argumentos}

Existen distintas herramientas para la construcción de mapas de argumentos; en este caso, nos referiremos a Debategraph, un recurso de código abierto para la construcción colaborativa de argumentos que combina la cartografía y la edición basada en el concepto de Wiki ${ }^{3}$. Desde el punto de vista argumentativo, la idea principal que subyace al recurso es la de representar todos los puntos de vista relevantes en el marco de un problema y, en función de esto, resolver diferencias de opinión y buscar soluciones a dicha cuestión (VAN EEMEREN; GROOTENDORST; HENKEMANS, 2006).

En este marco, se utilizó la herramienta Debategraph para plantear los principales argumentos en torno a diferentes problemáticas inherentes al ingreso y la permanencia en carreras universitarias en Argentina. Entre los resultados más relevantes, los estudiantes de profesorado de Biología señalaron que la posibilidad de participar en la construcción de mapas de argumentos permitió: conocer los argumentos de los autores de referencia $(68 \%)$, conocer la opinión de los compañeros de cursado (57\%), y llegar a acuerdos con los compañeros de cursado (41\%). En cuanto a los aspectos negativos, los futuros profesores señalaron que resulta difícil acordar el peso que se le debe asignar a cada argumento $(38 \%)$ y resolver diferencias de opinión con los compañeros cursado (27\%).

De este modo, la búsqueda de un consenso unánime se trasforma en un elemento positivo para algunos alumnos y negativo para otros. Así, tal como plantean Elam y Bertilsson (2003), dicha búsqueda puede significar la exclusión de puntos de vistas, una opción intermedia, desde una perspectiva más democrática, podría incluir la promoción de un consenso de carácter conflictivo y discutible, en el cual la disidencia sea un valor y, por lo tanto, se brinde el espacio para que ésta se manifieste.

Por otro lado, al igual que los foros, los mapas de argumentos se constituyen en recursos de valor para la formación del profesorado en torno a temáticas socio-científicas: orientados por un formador, los docentes y futuros docentes pueden aprender sobre diferentes temáticas y pensar con qué contenidos y basándose en qué fuentes de información podrían implementar un recurso como este con sus propios estudiantes.

\footnotetext{
${ }^{3}$ Disponible en: <http://debategraph.org/>. Visitado en: 5 oct. 2016.
} 
En la Figura 1, puede observarse un mapa de argumentos que reconstruye los aportes de distintos profesores de Biología y Química respecto del cultivo de plantas transgénicas en Argentina, en este caso, haciendo énfasis en un tema en particular: la deforestación.

Es importante decir que tanto profesores como estudiantes pueden construir mapas de argumentos tomando como referencia los debates asíncronos realizados en foros, transformándose así en herramientas de seguimiento del progreso de los estudiantes y de metacognición. Además, los mapas elaborados por un grupo de estudiantes podrían tomarse como elemento de análisis por parte de otros grupos de estudiantes para así avanzar en la comprensión de un determinado contenido. Finalmente, los cursos o instancias de formación docente inicial o continua podrían tomar como insumo mapas de argumentos elaborados por distintos grupos de alumnos o docentes para analizar los argumentos centrales presentados, los argumentos ausentes y el modo en que se relacionan las ideas, entre otras cuestiones.

Figura 1. Mapa de argumentos en torno al tema de la deforestación en el marco del cultivo de plantas transgénicas en Argentina

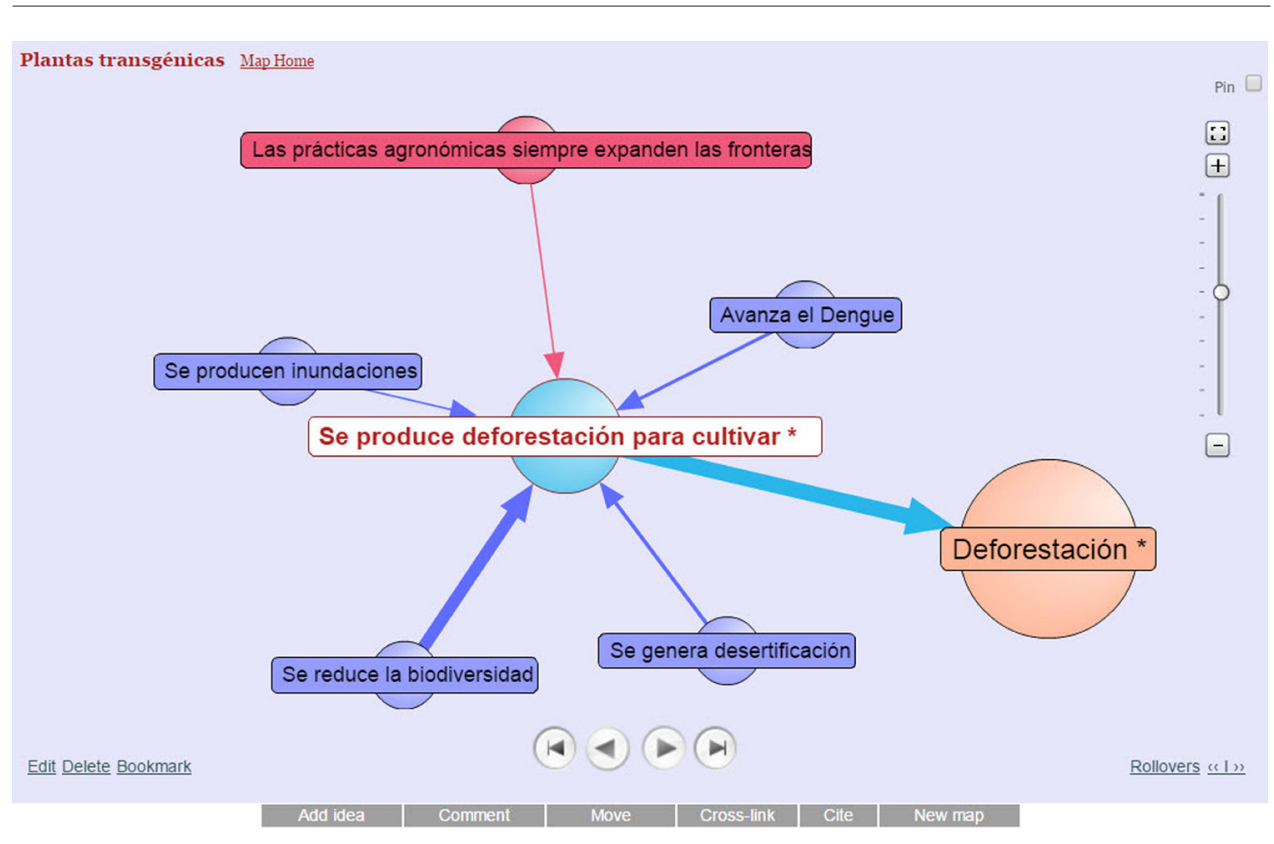

Fuente: elaborado por las autoras a partir de la página Web: debategraph.org

\section{Entorno WISE}

WISE es una plataforma digital de aprendizaje de las ciencias basada en el modelo didáctico por indagación creada por un equipo de la Universidad de Berkeley, California, que 
está en funcionamiento desde el año 19964. En este marco, el Proyecto SABIO ha traducido y adaptado parte de los proyectos allí presentes para la comunidad hispanoparlante (RIZZI IRRIBARREN et al., 2014).

WISE se organiza basándose en proyectos que proponen construir hipótesis, buscar información y criticar puntos de vista, entre otras cuestiones. Para lograr dichos objetivos, cada proyecto incorpora simulaciones, herramientas para realizar gráficos, herramientas de modelización, enlaces a otras páginas Web, etc. (LINN; CLARCK; SLOTTA, 2003; ROMERO ARIZA; QUESADA ARMENTEROS, 2014). En lo que atañe al plano argumentativo, el entorno se basa fundamentalmente en la noción de argumentación como evaluación de enunciados a la luz de las pruebas (JIMÉNEZ-ALEIXANDRE, 2010) y con actividades que otorgan distinta prioridad a la persuasión. Además, se incluye la posibilidad de trabajar colaborativamente.

Un aspecto central para la formación de profesores es que, como señalan Romero Ariza y Quesada Armenteros (2014), cualquier profesional interesado en la generación de recursos para la enseñanza de las ciencias puede agregar nuevos proyectos de investigación o realizar propuestas de mejora a los ya existentes.

En nuestro caso particular, el diseño con WISE se llevó a cabo en el marco de la formación inicial de profesores de ciencias, proponiéndoles a los futuros docentes evaluar diferentes proyectos del entorno a través de la construcción de una serie de dimensiones de análisis. En lo que se refiere a la dimensión argumentativa, los participantes pudieron caracterizar tanto el nivel de dificultad inherente a cada propuesta como la noción de argumentación que predominaba en el proyecto, permitiéndoles seleccionar el más adecuado para un grupo determinado de alumnos. Luego de esto, pudieron analizar los intercambios argumentativos de los alumnos tomando como base las ideas de Toulmin (SASSERON; CARVALHO, 2011; TOULMIN, 2007). Así, los futuros docentes desarrollaron herramientas de análisis esenciales que podrán utilizar más adelante para evaluar sistemáticamente otros recursos áulicos, pudieron vincular la dimensión argumentativa y tecnológica en vistas a su futura práctica docente, y redimensionaron el valor de la comunicación de docentes y alumnos y de alumnos entre sí.

\section{Consideraciones finales}

El presente trabajo abordó las creencias sobre argumentación de docentes y estudiantes, analizando sus características en términos de obstáculos y potencialidades para avanzar en la enseñanza de las ciencias. Además, se caracterizaron las concepciones de argumentación subyacentes a tres recursos tecnológicos particulares, resaltando sus potencialidades didácticas.

En lo que respecta a las creencias de los docentes y de los estudiantes, se hace hincapié en la complejidad de las nociones construidas en torno a la argumentación, las cuales convocan a pensar qué dimensiones podrían ser incorporadas a la formación docente en lo que respecta al tema. En este marco, se sostiene que dicha formación debería incluir diversas corrientes teóricas sobre la argumentación (y su relación con las creencias de los propios docentes), debería permitir

\footnotetext{
${ }^{4}$ Disponible en: <https://wise.berkeley.edu/>. Visitado en: 5 oct. 2016.
} 
a los profesores argumentar y construir actividades para que sus alumnos argumenten, debería incorporar el análisis de argumentos en contextos naturales de enseñanza, y debería incluir el debate respecto de qué niveles de argumentación queremos alcanzar con nuestros estudiantes.

En lo que se refiere a los recursos tecnológicos implicados, puede decirse que los tres combinan dos aspectos clave para el aprendizaje en ciencias: la argumentación y la colaboración. Además, presentan potencialidades para aprender a argumentar y para aprender ciencias a través de la argumentación. En lo que respecta a la formación de docentes de ciencias con tecnologías, las concepciones de argumentación subyacentes a cada recurso deberían volverse explícitas, y las herramientas o aplicaciones tecnológicas deberían utilizarse para argumentar pero también para que los docentes nos aproximemos y comprendamos mejor las prácticas argumentativas de los estudiantes.

Finalmente, y en consideración de las proyecciones de la línea de investigación, se sostiene que es necesario indagar las creencias sobre argumentación de docentes que atraviesan instancias de formación docente sobre la temática, estudiar las ideas de argumentación que subyacen a otras aplicaciones tecnológicas para aprender ciencias naturales (laboratorios virtuales, simulaciones, etc.), y analizar la argumentación producida en contextos multimodales de enseñanza.

\section{Agradecimientos}

Se agradece al CONICET por la beca postdoctoral otorgada a la Dra. Leticia Garcia Romano y a SECyT-UNC por el financiamiento otorgado para la realización de la investigación.

\section{Referencias}

ADÚRIZ-BRAVO, A. Revisiting school scientific argumentation from the perspective of the history and philosophy of science. In: MATHEWS, M. R. (Ed.). International handbook of research in history, philosophy and science teaching. Dordrecht: Springer, 2014. p. 1443-1472.

ARCHILA, P. A. La investigación en argumentación y sus implicaciones en la formación inicial de profesores de ciencias. Revista Eureka sobre Enseñanza y Divulgación de las Ciencias, Cádiz, v. 9, n. 3, p. 361-375, 2012.

ARGENTINA. Ministerio de Educación. Plan nacional de educación obligatoria y formación docente - Resolución CFE N 188/12 - 5 de diciembre de 2012. Disponible en: <http://www.me.gov.ar/consejo/resoluciones/res12/188-12_01_01.pdf>. Visitado en: 5 may 2013.

ARMSTRONG, S. L. Using metaphor analysis to uncover learners' conceptualizations of academic literacies in postsecondary developmental contexts. The International Journal of Learning, Melbourne, v. 15, n. 9, p. 211-218, 2008. 
BUTY, C.; PLANTIN, C. L'argumentation à l'épreuve de l'énseignement des sciences et viceversa. In: __ Argumenter en classe de sciences: du débat à l'apprentissage. Lyon: Institut National de Recherche Pédagogique, 2008. p. 17-41.

CAMPANER, G. La argumentación en la formación inicial de profesores de biología. In: AMIDOLARE, P. (Ed.). Memorias de las VIII Jornadas Nacionales y III Congreso Internacional de Enseñanza de la Biología. Mar del Plata: ADBiA, 2008. p. 388-391.

CARLINO, P. Enseñar a escribir en la universidad: cómo lo hacen en Estados Unidos y por qué. Revista Iberoamericana de Educación, Madrid, [2002]. Disponible en: <http:// rieoei.org/deloslectores/279carlino.pdf>. Visitado en: 16 jul. 2008.

CLARK, D. B. et al. Technology-enhanced learning environments to support students' argumentation. In: ERDURAN, S.; JIMÉNEZ-ALEIXANDRE, M. P. (Ed.).

Argumentation in science education: perspectives from classroom-based research. Dordrecht: Springer, 2008. p. 217-244.

DE VRIES, E.; LUND, K.; BAKER, M. Computer-mediated epistemic dialogue: explanation and argumentation as vehicles for understanding scientific notions. The Journal of the Learning Sciences, Abingdon, v. 11, n. 1, p. 63-103, 2002.

DORI, Y. J.; SASSON, I. Chemical understanding and graphing skills in an honors casebased computerized chemistry laboratory environment: the value of bidirectional visual and textual representations. Journal of Research in Science Teaching, Hoboken, v. 45, n. 2, p. 219-250, 2008.

DRIVER, R.; NEWTON, P.; OSBORNE, J. Establishing the norms of scientific argumentation in classrooms. Science Education, Hoboken, v. 84, n. 3, p. 287-312, 2000.

ELAM, M.; BERTILSSON, M. Consuming, engaging and confronting science: the emerging dimensions of scientific citizenship. European Journal of Social Theory, New York, v. 6, n. 2, p. 233-251, 2003.

GARCIA ROMANO, L.; OCCELLI, M. Argumentar en la formación profesional continua: un curso de capacitación para docentes de biología y química. RILL Nueva Época, San Miguel de Tucumán, v. 7, n. 1/2, p. 73-83, 2012.

GARCIA ROMANO, L.; VALEIRAS, N. Leer y escribir en un entorno digital: prácticas innovadoras en la carrera de ciencias biológicas. Arbor, Madrid, v. 187, n. extra 3, p. 285-289, 2011. Disponible en: < http://arbor.revistas.csic.es/index.php/arbor/article/ viewArticle/1441>. Visitado en: 6 oct. 2016.

GARCIA ROMANO, L. et al. Las tecnologías de la información y la comunicación en la formación docente continua de profesores de ciencias. In: PINILLA, A. I. A.; MARCO, J. L. A. (Ed.). Actas de las Jornadas Virtuales de Colaboración y Formación Virtual USATIC. Madrid: Bubok Publishing, 2015.

JIMÉNEZ-ALEIXANDRE, M.P. Diez ideas clave: competencias en argumentación y uso de pruebas. Barcelona: Graó, 2010. 
JIMÉNEZ-ALEIXANDRE, M. P.; ERDURAN, S. Argumentation in science education: an overview. In: _. Argumentation in science education: perspectives from classroombased research. Dordrecht: Springer, 2008. p. 3-27.

. Argumentation. In: GUNSTONE, R. (Ed.). Encyclopedia of science education. Dordrecth: Springer, 2015. p. 54-59.

KELLY, G. J.; BAZERMAN, C. How students argue scientific claims: a rhetorical-semantic analysis. Applied Linguistics, Oxford, v. 24, n. 1, p. 28-55, 2003.

LAKOFF, G.; JOHNSON, M. Metaphors we live by. Chicago: The University of Chicago Press, 1980.

LARRAÍN, A. El rol de la argumentación en la alfabetización científica. Estudios Públicos, Santiago de Chile, v. 116, n. 4, p. 167-193, 2009.

LEITÃO, S. La dimensión epistémica de la argumentación. In: KRONMÜLLER, E.;

CORNEJO, C. (Ed.). Ciencias de la mente: aproximaciones desde Latinoamérica. Santiago de Chile: J C Sáez Editor, 2007. p. 5-32.

LEVIN, T.; WAGNER, T. In their own words: understanding student conceptions of writing through their spontaneous metaphors in the science classroom. Instructional Science, Dordrecht, n. 34, p. 227-278, 2006.

LINEAMIENTOS básicos sobre formación docente de profesores universitarios. San Juan: ANFHE-CUCEN, 2011. Disponible en: < http://www.anfhe.org.ar/archivos/lineas_trabajo/ documento_comision_mixta\%20anfhe-cucen.pdf $>$. Visitado en: 5 oct. 2016.

LINN, M.; CLARCK, D.; SLOTTA, J. D. WISE design for knowledge integration. Science Education, Hoboken, v. 87, n. 4, p. 517-538, 2003.

LUEHMANN, A.; FRINK, J. Web 2.0 technologies, new media literacies, and science education: exploring the potential to transform. In: FRASER, B. J.; TOBIN, K.;

MCROBBIE, C. J. (Ed.). Second international handbook of science education: part two. Dordrecht: Springer, 2012. p. 823-838.

MARTON, F. Phenomenography: describing conceptions of the world around us.

Instructional Science, Amsterdam, v. 10, n. 2, p. 177-200, 1981.

MATEOS, M.; SOLÉ, I. Undergraduate students' conceptions and beliefs about academic writing. In: CASTELLÓ, M.; DONAHUE, C. (Ed.). University writing: selves and texts in academic societies. London: Emerald, 2012. p. 53-67.

OCCELLI, M.; GARCIA, L.; MASULLO, M. Integración de las TICs en la formación inicial de docentes y en sus prácticas educativas. VEC: virtualidad, educación y ciencia, Córdoba, v. 3, n. 5, p. 53-72, 2012. Disponible en: <https://revistas.unc.edu.ar/index.php/vesc/ article/view/3013/2868>. Visitado en: 5 oct. 2016.

PAREDES, J; ARRUDA, R. D. La motivación del uso de las TIC en la formación de profesorado en educación ambiental. Ciência \& Educação, Bauru, v. 18, n. 2, p. 353-368, 2012. Disponible en: <http://www.scielo.br/pdf/ciedu/v18n2/a08v18n2.pdf>. Visitado en: 5 oct. 2016. 
PEREIRA, C.; DI STEFANO, M. El taller de escritura en posgrado: representaciones sociales e interacción entre pares. Revista Signos, Valparaíso, v. 40, n. 64, p. 405-430, 2007. Disponible en: <http://www.scielo.cl/scielo.php?script=sci_ arttext\&pid=S0718-09342007000200007>. Visitado en: 5 oct. 2016.

PLANTIN, C. La argumentación. Barcelona: Ariel, 1998.

- Argumentar y manipular para probar. In: SOLER CASTILLO, S. (Ed.). Lenguaje y educación: perspectivas metodológicas y teóricas para su estudio. Bogotá: Universidad Distrital Francisco José de Caldas, 2012. p. 105-129.

. Lengua, argumentación y aprendizajes escolares. TED: tecné, episteme y didaxis, Bogotá, n. 36, p. 95-114, 2014. Disponible en: <http://www.scielo.org.co/pdf/ted/n36/ n36a07.pdf>. Visitado en: 5 oct. 2016.

. Pensar el debate. Revista Signos, Valparaíso, v. 37, n. 55, p. 121-129, 2004. Disponible en: <http://dx.doi.org/10.4067/S0718-09342004005500010>. Visitado en: 5 oct. 2016.

REVEL CHION, A. F. R.; MEINARDI, E.; ADÚRIZ-BRAVO, A. La argumentación científica escolar: contribución a la comprensión de un modelo complejo de salud y enfermedad. Ciência \& Educação, Bauru, v. 20, n. 4, p. 987-1001, 2014. Disponible en: <http://dx.doi.org/10.1590/1516-73132014000400014>. Visitado en: 5 oct. 2016.

RIZZI IRRIBARREN, C. et al. Diseño e implementación de la plataforma virtual de aprendizaje WISE en el aprendizaje de las ciencias naturales. In: CONGRESO IBEROAMERICANO DE CIENCIA, TECNOLOGÍA, INNOVACIÓN Y EDUCACIÓN, 2014, Buenos Aires. Memorias... Disponible en: <http://www.oei.es/ historico/congreso2014/30memorias2014.php>. Visitado en: 9 mayo 2015.

ROMERO ARIZA, M.; QUESADA ARMENTEROS, A. Nuevas tecnologías y aprendizaje significativo de las ciencias. Enseñanza de las Ciencias, Barcelona, v. 32, n. 1, p. 101-115, 2014.

SANDOVAL, W. A.; MILLWOOD, K. A. What can argumentation tell us about epistemology? In: ERDURAN, S.; JIMÉNEZ-ALEIXANDRE, M. P. (Ed.). Argumentation in science education: perspectives from classroom-based research. Dordrecht: Springer, 2008. p. 71-88.

SASSERON, L. H.; CARVALHO, A. M. P. Construindo argumentação na sala de aula: a presença do ciclo argumentativo, os indicadores de alfabetização científica e o padrão de Toulmin. Ciência \& Educação, Bauru, v. 17, n. 1, p. 97-114, 2011. Disponible en: < http:// dx.doi.org/10.1590/S1516-73132011000100007>. Visitado en: 5 oct. 2016.

SCARDAMALIA, M.; BEREITER, C. Dos modelos explicativos de los procesos de comprensión escrita. Infancia y Aprendizaje, Madrid, v. 15, n. 58, p. 43-64, 1992.

SOLANO ARAUJO, I.; VEIT, E. A.; MOREIRA, M. A. Physics students' performance using computational modelling activities to improve kinematics graphs interpretation.

Computers \& Education, Amsterdam, v. 50, n. 4, p. 1128-1140, 2008. 
TOULMIN, S. Los usos de la argumentación. Barcelona: Península, 2007.

VAN EEMEREN, F.; GROOTENDORST, R.; HENKEMANS, F. S. Argumentación: análisis, evaluación, presentación. Buenos Aires: Biblos, 2006.

VÁZQUEZ-ABAD, J. et al. Fostering distributed science learning through collaborative technologies. Journal of Science Education and Technology, Dordrecht, v. 13, n. 2, p. 227-232, 2004.

ZANOTTI, A.; ARANA, A. Implementación del Programa Conectar Igualdad en el aglomerado Villa María-Villa Nueva, Córdoba, Argentina. Ciencia, Docencia y Tecnología, Concepción del Uruguay, v. 26, n. 50, p. 120-143, 2015.

ZOHAR, A. Science teacher education and professional development in argumentation. In: ERDURAN, S.; JIMÉNEZ-ALEIXANDRE, M. P. (Ed.). Argumentation in science education: perspectives from classroom-based research. Dordrecht: Springer, 2008. p. $245-268$.

Artigo recebido em 05/11/2015. Aceito em 30/01/2016.

Dirección para contacto: Universidad Nacional de Córdoba, Departamento de Enseñanza de la Ciencia y la Tecnología, Avenida Vélez Sarsfield 299, Córdoba 5000, Argentina 